\title{
Pygmalions contemporains ou la nouvelle Galatée dans À ciel ouvert de Nelly Arcan
}

\author{
Pascale Joubi \\ Université de Montréal
}

Implicitement présente derrière la construction et la représentation des rapports de force traditionnels entre le masculin et le féminin, la figure mythologique de Pygmalion se fait une métaphore du pouvoir de l'homme sur la femme: comme le déclarent Christine Détrez et Anne Simon, auteures d'À leur corps défendant (2006), dans ce mythe, «[1]'homme fait la femme, et la dote de sa féminité » (p. 52), il «l'épanoui[t] sexuellement» (p.53) et lui «attribue l'identité » (p. 53). Mais la littérature n'est plus le seul lieu d'accueil de figures porteuses de cette idéologie. À l'époque contemporaine, avec l'épanouissement de la chirurgie plastique, des figures de 
Pygmalion et de Galatée au sens « littéral » du mythe sont nées : le chirurgien esthétique et son œuvre, une femme en chair et en os, remodelée selon les impératifs de beauté de l'heure. À travers la mise en scène d'un triangle amoureux classique et le ressassement de thématiques telles que la tyrannie de l'industrie de la beauté, la sexualité et les relations interpersonnelles malsaines, qui lui sont chères - parce qu'elles définissent, à ses yeux, la société nord-américaine, pour ne pas dire occidentale, contemporaine -, Nelly Arcan offre dans $\grave{A}$ ciel ouvert une critique des jeux de pouvoir traditionnels entre les sexes, critique qu'il est possible de relever par l'étude de la réappropriation par l'auteure du mythe de Pygmalion et de Galatée. Chez Arcan, deux personnages masculins réincarnent le sculpteur: d'un côté, Charles, l'(ex-)amant, photographe de mode, crée des Galatées virtuelles parfaites, exposées sur les pages des magazines et les affiches publicitaires; d'un autre côté, le Dr Gagnon, chirurgien esthétique, sculpte des femmes réelles en des Galatées à l'image des modèles que Charles photographie et perfectionne par Photoshop. La protagoniste du récit, Rose, ne peut échapper à l'influence et au désir de ces deux Pygmalions contemporains. Cependant, - et là se situe le point fort de la réécriture du mythe par Arcan -, si Rose se plie aux attentes de ces personnages, elle les pousse à leur limite afin de gagner en subjectivité et en pouvoir, dévoilant, derrière le masque de Galatée, une véritable Médée.

\section{Pygmalions et Galatées contemporains}

À sa première rencontre avec Rose, Julie, le deuxième personnage féminin d'À ciel ouvert, décrit celle qui deviendra à 
la fois sa rivale et sa complice : "Cette femme [est] vraiment belle mais d'une façon commerciale, industrielle, [note-t-elle] sans la juger puisqu'elle en [fait] elle-même partie, de cette famille de femmes dédoublées, des affiches. » (p. 15) Ces filles en série ${ }^{1}$ sont exposées dans les revues de mode, dans les médias et sur les sites Internet après avoir été fabriquées par le discours contemporain sur le corps stigmatisé par la chirurgie esthétique ${ }^{2}$ et par le travail de l'image qui cherche à traduire ce discours en montrant des modèles vivants, retouchés pour correspondre aux standards de beauté de leur temps. Ainsi, «Charles, dans À ciel ouvert, devient "l'instrument de mesure", l'homme qui, par le biais de l'appareil photographique, crée le canon, la norme à respecter en matière de beauté féminine » (Dugas, 2010, p. 48). En d'autres mots, le photographe produit des Galatées virtuelles grâce aux miracles de Photoshop, l'outil principal du sculpteur moderne qu'incarne Charles. Là où Pygmalion perfectionne sa statue d'ivoire, Charles rend la peau de ses modèles aussi lisse et brillante que la surface de l'image qui les enferme. Étant quotidiennement confrontées à ces images, les femmes «réelles» de l'univers arcanien ressentent de la pression pour devenir conformes au modèle de la femme idéale vendu dans les médias et la publicité. L'industrie de la beauté vient à leur secours en leur offrant produits cosmétiques et techniques chirurgicales miraculeux accompagnés du slogan

\footnotetext{
1 «Les filles en série sont ces jumelles dont les mouvements s'agencent parfaitement, qui bougent en harmonie les unes aux côtés des autres, qui ne se distinguent les unes des autres que par le détail d'un vêtement, de chaussures, d'une teinte de cheveux ou de peau, par des courbes légèrement dissemblables... Filles-machines, filles-images, filles-spectacles, fillesmarchandises, filles-ornements... elles sont l'illusion de la perfection. » (Delvaux, 2013, p. 10-11; l'italique appartient à l'auteure.)

2 À propos de la représentation du corps dans À ciel ouvert, voir Dugas (2010) et Labrosse (2010).
} 
«Pour moi, moi, moi ${ }^{3}$ (Arcan, 2007, p. 95). Ces messages publicitaires ont, en vérité, un effet pervers, créant l'illusion que les interventions esthétiques sont des «cadeaux faits à soimême et pour soi-même » (Arcan, 2007, p. 95). Ils masquent ainsi ce qu'Arcan dénonce dans l'œuvre par le truchement des protagonistes : le recours à la chirurgie plastique s'avère une soumission aux "normes esthétiques hétérosexistes » (Dugas, 2010, p.49). Afin de plaire aux personnages masculins, les personnages féminins d'À ciel ouvert subissent la douleur qui accompagne leur "acharnement esthétique » (Arcan, 2007, p. 89). Et la séduction par le corps paraît, à première vue, être l'unique façon que les personnages féminins d'Arcan ont trouvée pour obtenir du pouvoir.

Leur complice, dans la mesure où il leur permet d'accéder à cette forme de pouvoir tout en faisant partie de l'industrie qui les soumet à des normes hétérosexistes ${ }^{4}$, est le deuxième type de Pygmalion, le chirurgien esthétique. Figure moderne d'un mythe ancien, il sculpte le corps des femmes de façon qu'il réponde aux normes de beauté instaurées dans les médias, fabriquées par les artisans de Photoshop et l'industrie de la mode. La profession même transpose la division genrée des rôles qui se trouve dans le mythe : les chirurgiens, souvent des hommes, ont le rôle actif, ils opèrent les femmes qui sont des objets modulables, comme Galatée.

\footnotetext{
${ }^{3}$ En italique dans le texte.

${ }^{4}$ Dugas relève à juste titre le paradoxe, noté d'ailleurs par Rose elle-même (Arcan, 2007, p. 120-121), dans cette forme de pouvoir : «Les personnages de Rose et de Julie se définissent à l'aune de leur pouvoir de séduction. La chirurgie esthétique devient pour elles un moyen d'accéder à "l'autonomie" que procure une enveloppe charnelle répondant aux normes. Il demeure que dans [À ciel ouvert] de Nelly Arcan, le paradoxe entre l'état subjectal et objectal des personnages féminins est mis de l'avant. » (p. 27)
} 
Effectivement, dans $\grave{A}$ ciel ouvert, Julie note que le corps de Rose «porte une signature» (Arcan, 2007, p. 120). Le corps retravaillé par le Dr Gagnon devient une œuvre d'art que l'artiste signe pour en réclamer le droit d'auteur, et les personnages féminins d'Àciel ouvert, surtout Rose, semblent appartenir autant au chirurgien qu'au photographe, car elles ont recours au premier pour atteindre l'idéal du second et obtenir un corps qui lui arrachera le plaisir sexuel. Cette recherche d'idéal est la prémisse du mythe de Pygmalion, fixé par Ovide dans Les Métamorphoses. L'excellent sculpteur, dégoûté par « les vices que la nature a prodigalement départis à la femme » (Ovide, 1966, p. 260), choisit le célibat, préférant passer son temps à créer une statue d'ivoire et à lui «donn[er] une beauté qu'aucune femme ne peut tenir de la nature » (p. 260). Chez Arcan, l'idéal féminin correspond à ce que Julie appelle les «femmes-vulves », c'est-àdire « des femmes entièrement recouvertes de leur propre sexe » (p. 90), d'une burqa de chair. Cette « burqa occidentale » (p. 89) est tissée à même le corps des femmes par la chirurgie esthétique: elles se trouvent prises au piège dans un corps devenu objet malléable, modulable selon les standards de beauté de la société contemporaine, comme la Galatée du mythe est enfermée dans son statut de sujet-objet. Effectivement, lorsque, par le pouvoir de Vénus exauçant le vœu d'un Pygmalion épris de sa « vierge d'ivoire » (Ovide, 1966, p. 261), Galatée se transforme en femme de chair et d'os, elle demeure traitée comme un objet d'art. Chez Ovide, la seule action qu'elle pose, ou plutôt la seule réaction qui lui est attribuée, c'est de « rougir » avant de «lev[er] timidement son regard » (p. 261) pour voir « en même temps que le ciel [...] celui qui l'aimait» (p. 261). Cette hiérarchie dans les rôles est maintenue lors de la recréation de la femme, et les réécritures de Pygmalion ou les œuvres ultérieures aux 
Métamorphoses qui s'en sont inspirées reconduisent la double thématique de l'animé/inanimé, du sujet/objet. Toutefois, les personnages féminins d'À ciel ouvert, Rose en particulier, ne sont pas uniquement le bel objet d'art du mythe originel : leur beauté se conjugue à une monstruosité ${ }^{5}$ née du brouillage des limites entre le naturel et l'artificiel dont témoignent les quasi invisibles cicatrices laissées par la chirurgie esthétique. À vrai dire, le recours aux opérations plastiques place les protagonistes du récit dans une position de précarité : un faux pas, une erreur de la part du chirurgien, ou simplement le passage du temps, et elles pourraient se retrouver «du côté des monstres » (Arcan, 2007, p. 183). Mais c'est délibérément que Rose, animée par un esprit de vengeance, par la volonté de punir Charles qui l'a trompée avec Julie, franchit la frontière en subissant une vaginoplastie.

\section{Monstration}

CEuvre de Charles et du Dr Gagnon, Rose-Galatée se fait miroir de la monstruosité des personnages masculins, devenant, à la fin du récit, davantage parente de l'effrayante Méduse que de la poupée taille nature qu'elle était au début d'À ciel ouvert ${ }^{6}$. Étymologiquement, le substantif «monstre» vient du verbe

\footnotetext{
${ }^{5}$ Je propose la définition suivante du monstrueux, tout en admettant que sa simplicité est loin de rendre compte de la complexité de cette notion, que je développe en détail dans Joubi, 2014, p. 23-31: Par définition, le monstrueux désigne ce qui dépasse les normes et les limites de la différence acceptable, provoquant le plus souvent un sentiment d'attraction/répulsion chez celui qui regarde.

${ }^{6}$ « [Après la vaginoplastie], Rose n'était plus tout à fait Rose. L'illusion de sa petitesse fragile à secouer à bout de bras, à faire voler dans les airs, ne pouvait plus tenir. Rose était redoutable de volonté, elle ne pouvait pas être petite; pendant tout ce temps c'est elle qui avait été la meneuse. »(Arcan, 2007, p. 200)
} 
latin monstrare, qui veut dire montrer. Faire voir l'a-normal, l'exposer, l'exhiber est donc le propre du monstrueux. Parfois même, la monstruosité réside davantage dans l'exhibition du monstre que dans son existence même, car, une fois sorti au grand jour, le monstre ne peut plus être ignoré. Rose, une fois sur le toit de l'immeuble où les personnages sont réunis pour le shooting d'un documentaire, alors qu'elle s'apprête à exhiber son nouveau sexe comme on exposait les monstres dans les foires et les cirques de la fin du XIXe siècle, déclare : «Toi aussi Charles! Viens voir encore plus près l'endroit où les hommes comme toi poussent les femmes! » (Arcan, 2007, p. 248) Ce sont en partie les goûts déviants de son (ex-)amant Charles en matière sexuelle qui expliquent sa décision de subir une vaginoplastie. Surnommé «l'équarrisseur», le photographe n'est excité que par la vue de morceaux de corps bien précis, et si ces morceaux sont marqués par la blessure, par l'ecchymose et par la cicatrice-notamment celle issue d'une chirurgie plastique -, sa jouissance en sera intensifiée. En ayant recours à la vaginoplastie, Rose va à la rencontre de la monstruosité de Charles, pour lui offrir le sexe idéal dont il rêve. Alors, à ce moment du récit, Arcan effectue un déplacement de la monstruosité du corps retravaillé à l'être qui a contribué à le créer, le personnage masculin abject par ses goûts sexuels qui s'érigent en impératifs auxquels les personnages féminins se sentent obligés de se plier. Rose fait donc porter à Charles la responsabilité de sa transformation en monstre. En même temps, passer du côté du monstre est pour Rose-Galatée une forme de prise de pouvoir : en faisant remodeler son sexe par le Dr Gagnon 7 , elle croit enfin réussir à garder pour elle, grâce à la

\footnotetext{
${ }^{7}$ Plus le récit avance, plus le docteur Gagnon perd son pouvoir sur Rose : celle-ci réussit, en jouant le rôle de la femme docile, à obtenir de lui des
} 
puissance de son sexe recréé, son amant, qui ne peut se donner qu'à des morceaux de corps féminin. Marqué par les traits de l'insolite et de l'étrange, son sexe, une fois opéré, altéré, abjecté, devient un objet monstrueux ${ }^{8}$ : "C'était un sexe nu, qui n'avait plus de peau parce que la mort l'avait soulevée [...]. » (Arcan, 2007, p. 216) Le pouvoir mortifère de ce sexe est mis en évidence, comme une prophétie de ce qui adviendra à la fin du récit, le suicide de Charles qui se jette du toit de son immeuble à la suite de la vue du nouveau sexe de Rose. «Voir la mort, c'est mourir, par contagion mimétique instantanée » (p. 214), affirme Françoise Frontisi-Ducroux à propos de Méduse. Si la Galatée mythologique est porteuse de vie, son avatar moderne présenté par Arcan s'apparente finalement à une figure mortifère : Rose se fait Méduse par son sexe-monstre. Cette parenté lui procurera, ne serait-ce que momentanément, une position de pouvoir et un accès au statut de sujet.

traitements gratuits et un gîte lorsqu'elle quitte Charles, et le manipule au point de lui faire accepter d'opérer son sexe. D'ailleurs, du moment où le $\mathrm{Dr}$ Gagnon procède à la vaginoplastie, il perd Rose, son œuvre, et se retrouve dans la filiation des Pygmalions dépassés par leur propre création (Arcan, 2007, p. 200-201).

${ }^{8}$ On peut se demander si, dans un univers comme celui qu'Arcan met en scène, où la chirurgie esthétique paraît comme une nouvelle norme à suivre, le monstrueux issu des opérations plastiques n'est pas normalisé tant il aura été répandu et montré. Si les interventions chirurgicales et plastiques sur un corps humain semblent banales parce que pratiquées sur presque tous les personnages féminins mis en scène, la monstruosité ressurgit, entre autres, lorsque les interventions témoignent d'un excès, comme dans le cas de la vaginoplastie de Rose, ou lorsque des actes jugés "moralement inadmissibles » sont commis. Dans les deux cas, les personnages concernés s'attirent beaucoup moins d'indulgence qu'on ne penserait dans le microcosme mis en scène dans À ciel ouvert, où la norme, telle qu'elle est définie au début du XXIe siècle dans la société occidentale, sert toujours de règle de mesure de la transgression des limites qu'elle impose. 


\section{De Galatée à Méduse}

Le sexe de Rose n'est pas monstrueux simplement parce qu'il est artificiel : ce qui le rend aussi abject, c'est l'œil que Charles, le photographe, y voit : «Au centre de la chair s'ouvrait un œil, non pas l'image d'un œil, non pas un œil photographié, mais un vrai, un œil vivant [...]. [...] l'œil l'avait regardé droit dans son œil à lui, intentionné, prêt à lui parler, sans malice ». (Arcan, 2007, p. 237) Par le biais de cet œil-sexe, Rose-Galatée devient méduséenne, puisque le thème central du mythe de Méduse est « celui de l'œil, du regard, de la réciprocité du voir et de l'êtrevu » (Vernant, 1998, p. 77). Regarder quelqu'un dans les yeux, c'est aller à la rencontre de soi, ou de l'Autre en soi, à travers la vue de son propre reflet dans la pupille de l'autre. Regarder Méduse revient à constater «l'horreur terrifiante d'une altérité » (Vernant, 1998, p. 84) qui pétrifie celui qui regarde parce qu'elle le force à s'y identifier. Que voit donc Charles dans l'œil-sexe? Au lieu de voir Rose et d'en être consumé de désir, il voit son père, «le Père Boucher » (Arcan, 2007, p. 273), et, par extension, sa déviance, ses goûts monstrueux, qui «l'horrifiaient lui-même» (Arcan, 2007, p. 106), qui sont «le contraire de [...] la nature » (Arcan, 2007, p. 106). Ce sexe, fait sur mesure pour lui plaire, réfléchit l'image de l'Autre terrifiant, parce qu'a-normal, caché en lui ${ }^{9}$, et le rend fou, littéralement. Ce qui provoque l'effroi et la pétrification (sans érection) dans À ciel ouvert n'est pas tant le sexe féminin, contrairement à ce que soutient Freud dans « La tête de Méduse » (p. 49), mais un

\footnotetext{
${ }^{9}$ L'Autre ici serait en fait le même, parce que Charles ne voit pas la mère dans le sexe de Rose, mais le père; il n'y voit pas la femme, altérité absolue aux yeux de sujet masculin « universel».
} 
désir excessif nourri d'images pornographiques ayant poussé une femme, Rose, à devenir une Galatée monstrueuse.

Méduse ne détient pas seulement le pouvoir de pétrification, mais possède aussi celui d'aveuglement. Dans À ciel ouvert, l'œil-sexe de Rose-Galatée guérit Charles de son aveuglement; le photographe, dès qu'il pose ses yeux sur le sexe de Rose, devient plutôt (clair)voyant. En octroyant ce pouvoir positif au sexe-monstre, Nelly Arcan opère, sur un autre plan, un renversement du mythe de Méduse, puisqu'elle transforme l'objet monstrueux en objet sacré10. Nelly Arcan procède à une sublimation du mythe de la Gorgone et donne à cette figure, et par son truchement à celle de la Galatée nouvelle, un grand pouvoir, un pouvoir certes mortifère, mais salutaire en ce sens que la lucidité que Charles acquiert en devenant (clair)voyant lui permet de s'élever vers l'au-delà s'il suit le chemin indiqué par le sexe de Rose, cette nouvelle voie du salut. Cependant, tel Icare qui tend vers le soleil avant sa chute brutale, Charles chutera et connaîtra le sort qui suit inévitablement la rencontre avec Méduse, la mort, non pas pour avoir regardé pendant trop longtemps le sexe-monstre, mais pour ne pas avoir suivi le chemin du sexe sacré de Rose11, «chef des Amazones » (Arcan, 2007, p. 249).

La Galatée contemporaine qu'est Rose au début du récit se métamorphose donc d'abord en Méduse, puis en chef des Amazones. C'est par la greffe de ces trois mythes féminins non

10 «C'est interdit! C'est sacré!» (Arcan, 2007, p. 241), s'écrie notamment Charles, à propos du sexe de Rose, devant lequel il se prosterne.

11 «Tu ne l'as pas écoutée! Tu ne l'as pas suivie! Tu vas le payer! » (Arcan, 2007,242 ), profère la voix «menaçante » de la mère de Charles, voix qui fait partie du chœur qu'il croit entendre tout au long de son délire. 
associés les uns aux autres dans la mythologie antique ${ }^{12}$ qu'est créé un personnage féminin qui réussit à mettre en échec un personnage masculin. Galatée ne reste pas sage et passive dans À ciel ouvert: Rose, qui semble à première vue répéter des schémas hétérosexistes en reflétant dans ses actes la monstruosité inhérente, chez Arcan, au masculin, amène le discours dominant, qui a construit lesdits schémas, à s'autocritiquer.

En effet, dans le chapitre final du récit, Arcan opère un renversement complet des rôles sexués traditionnels dans le couple composé de Charles et de Rose à travers la figure du père de Charles. La voix de ce dernier sort de l'œil-sexe, alors que son fils l'observe, et proclame le contraire de ce qu'il proférait pendant l'enfance du photographe: «Mon fils, je m'étais trompé. Les femmes ne sont pas nos ennemies. Rose, leur chef, est l'Amazone, la voie. » (Arcan, 2007, p. 237) Rose, par son œil-sexe, devenu sexe sacré, règne désormais sur une lignée de femmes fortes, dominatrices, auxquelles l'homme se doit d'obéir. Plus encore, le père ordonne à son fils de bien regarder les femmes comme Rose et à ne plus les craindre parce que leur « sexe sacré [...] mène à l'au-delà » (Arcan, 2007, p. 240). Lorsque Charles, complètement pétrifié, n'a pas su suivre la voie indiquée par le sexe de Rose, la voix de son père l'abandonne et «murmur[e] pour Rose [...] : “C'est bien, c'est bien, c'est bien"» (Arcan, 2007, p. 250). Le personnage masculin est alors exclu, rejeté, ab-jecté, par sa folie et par son rapport pathologique au sexe. Charles n'est donc plus exclusif, mais exclu : il occupe désormais une position de marginalité. Il

\footnotetext{
12 Il faut tout de même préciser que, dans certains textes anciens, Méduse et les Amazones ont été associées. Voir Garber et Vickers, 2003.
} 
n'est plus indestructible et infaillible, il est castré à la vue de l'œil-sexe de Rose, car l'érection consolatrice n'advient pas dans À ciel ouvert, contrairement aux attentes de Rose (Arcan, 2007, p. 240). Du moment où l'homme aussi est ab-jecté dans le récit, l'abjection du féminin prend un autre sens: la position de l'altérité n'est plus exclusivement occupée par la femme, qui la partage maintenant avec l'homme. Il semble donc que la réécriture des mythes de Galatée, de Méduse, des Amazones et de Pygmalion contribue à créer, dans À ciel ouvert, un univers où la femme serait l'égale de l'homme par le biais de l'abjection ${ }^{13}$ et à repenser les relations homme-femme tout en remettant en question, à travers la fiction, certaines représentations traditionnelles du sexe féminin, que celles-ci soient inspirées de Galatée, de la Gorgone ou des Amazones.

À la lumière de l'étude de la réécriture des mythes dans À ciel ouvert, il est possible de constater que l'obtention du pouvoir par le personnage féminin ne passe pas uniquement par l'aliénation de son corps aux goûts et aux attentes esthétiques des personnages masculins. Son pouvoir semble plutôt résider dans le fait que, se conformant à l'idéal instauré par les personnages, elle se fait miroir de la monstruosité des hommes de papier qu'elle côtoie ${ }^{14}$ ? Si au début du récit, Rose est une véritable Galatée, "se plaç[ant] d'elle-même en état de pur fétiche fait pour le sexe sans sexe » (Arcan, 2007, p. 107), à la fin, en atteignant un niveau de monstruosité reflétant celui de

13 Selon la vision du monde qu'Arcan propose dans ses œuvres, il est impossible à l'être humain d'échapper à l'abjection et, par le fait même, il lui est impossible de s'élever, de se sublimer. S'il y a égalité, ou pas, entre les sexes, elle sera toujours mesurée selon l'échelle de la bassesse, de l'abjection, de la monstruosité.

${ }^{14}$ Ce constat s'applique également au personnage principal de Folle (Arcan, 2004). Voir Joubi, 2014. 
Charles, Rose acquiert une capacité d'agir et réussit, par l'exhibition de sa « burqa de chair » (Arcan, 2007, p. 248) sur le toit de l'immeuble une véritable performance, une prise de parole qui lui a longtemps été refusée et qu'elle peut enfin récupérer en médusant son entourage. Quant aux Pygmalions du récit, le Dr Gagnon se fait manipuler et rejeter par celle qu'il a sculptée et Charles perd sa toute-puissance et paie de sa mort aux mains de sa créature le prix de sa monstruosité qui a engendré une Galatée nouvelle.

\section{Bibliographie}

ARCAN, Nelly. (2007), À ciel ouvert, Paris, Seuil, coll. « Points ».

—. (2004), Folle, Paris, Seuil, coll. « Points ».

DelvauX, Martine. (2013), Les Filles en série. Des Barbies aux

Pussy Riot, Montréal, Les Éditions du remue-ménage.

DÉTREZ, Christine et Anne Simon. (2006), À leur corps défendant: les femmes à l'épreuve du nouvel ordre moral, Paris, Seuil.

DuGAS, Marie-Claude. (2010), «Corps, identité et féminité chez Nelly Arcan et Marie-Sissi Labrèche », mémoire de maîtrise, Montréal, Université de Montréal.

FREUD, Sigmund. (1985 [1922]), "La tête de Méduse », dans Résultats, idées, problèmes, t. II : 1921-1938, Paris, Presses universitaires de France, p. 49-50.

FroNTISI-DUCROUX, Françoise. (2003), L'Homme-cerf et la femmearaignée. Figures grecques de la métamorphose, Paris, Gallimard.

GARBer, Marjorie et Nancy J. Vickers. (2003), The Medusa Reader, New York /Londres, Routledge. 
JouBI, Pascale. (2014), Mythes et monstres dans Folle et À ciel ouvert de Nelly Arcan, mémoire de maîtrise, Université de Montréal.

—. (2013), «L'œil de Méduse dans À ciel ouvert de Nelly Arcan », MuseMedusa, no 1 , <http://musemedusa.com/dossier 1/joubi/>.

LABRosSE, Claudia. (2010), «L'impératif de beauté du corps féminin : la minceur, l'obésité et la sexualité dans les romans de Lise Tremblay et de Nelly Arcan », Recherches féministes, vol. 23, no 2, p. 25-43.

Ovide (1966), Les Métamorphoses, traduction, introduction et notes par Joseph Chamonard, Paris, Garnier-Flammarion.

Vernant, Jean-Pierre. (1998), La Mort dans les yeux. Figures de l'Autre en Grèce ancienne. Artémis, Gorgô, Paris, Hachette Littératures.

\section{Résumé}

À ciel ouvert de Nelly Arcan propose une réécriture du mythe de Pygmalion et de Galatée à travers laquelle les catégories et les rôles du masculin et du féminin sont repensés. L'essor de la chirurgie esthétique et de la photographie numérique aux $\mathrm{XX}^{\mathrm{e}}$ et XXIe siècles permettent l'apparition de Pygmalions modernes sous la forme du chirurgien plasticien et du photographe, qui sculptent une Galatée propre à leur temps : une femme en chair et en os née du travail du scalpel et du curseur. Or, celle qui est mise en scène par Arcan ne demeure pas l'objet d'art du mythe originel: elle s'apparente davantage à l'effrayante Méduse et réussit ainsi à s'imposer à ses créateurs comme figure de pouvoir. 


\begin{abstract}
Nelly Arcan's À ciel ouvert offers a rewriting of Pygmalion and Galatea's myth through which the author rethinks the categories and roles of the masculine and the feminine. The development of plastic surgery and digital photography in the $20^{\text {th }}$ and $21^{\text {st }}$ centuries allowed the apparition of modern Pygmalions, like the plastic surgeon and the photographer, who sculpt a Galatea in the flesh. However, Arcan's Galatea isn't simply a female object of art: she shares some traits with the monstrous Medusa and thus become capable of dominating her creators.
\end{abstract}

